\title{
植物 学
}

\section{銀杏幼胚离体培养的研究}

\section{一一酪朊水解物、谷氨酰胺、天門冬酰胺、2,4-D及 硫酸腺尿園对幼胚生长及分化的影响}

\author{
王伏雄、李宪章 \\ (中国科学院植物研究所)
}

在幼胚及其他生殖器官的离体培养中, 酪朊水解物、谷氨酰胺和天门冬酰胺賏用作 生长辅助物质 ${ }^{[1]}$. 2,4-D 可诱导胚愈伤组织 的产生 ${ }^{[2]}$. 硫酸腺尿圆也㲘用于胚胎培养 ${ }^{[1]}$.

本文就酪朊水解物、谷氮酰胺、天门冬 酰胺、2,4-D 和硫酸腺尿圆对银杏幼胚生长 和分化的影响，作一简单报导.

银杏胚的解剖和消毒以及培养基的配制 和消毒同前一篇报告 ${ }^{[3]}$. 蔗糖用 $8 \%$ (小于 1,600 微米的胚)或 $5 \%(2,800-3,240$ 微米). 酪朊水解物用酪朊自制成 $10 \%$ 储备液。楖 子乳汁经蒸汽消毒.

培养肧置于恆温箱中 $\left(22 \pm 2{ }^{\circ} \mathrm{C}\right)$, 不加 光照. 每周检查记录一次. 试验结束时分別 照相及固定, 一部分材料制成制片观察.

\section{实 验 结 果}

\section{1. 酪朊水解物对幼胚生长的影响}

接种时肧分为两组,一组子叶倘未分化, 肧长 $1,080-1,390$ 微米; 另一组子叶已分 化, 肧长 1,280-1,620 微米, 培养 15 天后 的结果如表 1 所示.
从表 1 可以看出, 加入酪朊水解物 $(200$ $-1,600 \mathrm{ppm}$ )培养的胚, 都长得比对照的大, 而且接种时未分化的原胚, 增长百分比普遍 比已分化的胚要略高一些. 促进生长的作用 也表现于胚的全面生长. 在基本培养基上生 长的胚, 发育正常. 加入 $400 \mathrm{ppm}$ 酪朊水解 物培养的胚, 胚体较大. 在加入 800 及 1,600 $\mathrm{ppm}$ 酪朊水解物培养的胚, 一部分胚形成愈 伤组织, 胚体也显得更大些. 在 1964 年第 9 次试验中, 虽然酪朊水解物表现出促进幼胚 生长的明显作用, 却不产生愈伤组织.

接种时子叶已经长得较大的胚 $(2,800-$ 3,240 微米), 酪朊水解物的作用明显, 要求 较高的浓度 $(1,600-6,400 \mathrm{ppm})$.

根据制片观察表明, 加酪朊水解物培养 的胚, 內部构造正常, 可是发育速度比对照 的胚较快. 胚体较大, 显然是由于细胞数量 较多. 在子叶表面还可见分生组织区.

2. 谷氨酰胺及天门冬酰胺对幼胚生长的 影响

表 2 表示谷氨酰胺和天门冬酰胺对幼胚 ( $1,040-1,540$ 微米)生长的影响. 
表 1 酪朊水解物对幼胚生长的影响

1964 年第 3 次试验, 培养 15 天

\begin{tabular}{|c|c|c|c|c|}
\hline 养基 & 接种时肧长 (微米) & 压 数 & 接种时已否分化 & 15 天后增长 $(\%)$ \\
\hline 对照(WM) & $\begin{array}{l}1,210(1,080-1,320) \\
1,460(1,390-1,560)\end{array}$ & $\begin{array}{l}7 \\
5\end{array}$ & $\begin{array}{l}\text { 末 } \\
\text { 已 }\end{array}$ & $\begin{array}{l}89.3 \\
79.1\end{array}$ \\
\hline $\begin{array}{l}\text { WM }+200 p p m \text { 酪朊永解物 } \\
(\mathrm{CH})\end{array}$ & $\begin{array}{l}1,190(1,080-1,310) \\
1,460(1,380-1,620)\end{array}$ & $\begin{array}{r}10 \\
5\end{array}$ & $\begin{array}{l}\text { 未 } \\
\text { 巳 }\end{array}$ & $\begin{array}{l}110.0 \\
106.8\end{array}$ \\
\hline $\mathrm{WM}+400 \mathrm{ppmCH}$ & $\begin{array}{l}1,260(1,080-1,350) \\
1,450(1,360-1,620)\end{array}$ & $\begin{array}{l}12 \\
17\end{array}$ & $\begin{array}{l}\text { 未 } \\
\text { 已 }\end{array}$ & $\begin{array}{r}113.5 \\
96.6\end{array}$ \\
\hline $\mathrm{WM}+800 \mathrm{ppmCH}$ & $\begin{array}{l}1,160(1,080-1,230) \\
1,420(1,360-1,600)\end{array}$ & $\begin{array}{l}7 \\
8\end{array}$ & $\begin{array}{l}\text { ホ } \\
\text { 已 }\end{array}$ & $\begin{array}{l}118.9 \\
104.9\end{array}$ \\
\hline $\mathrm{WM}+1,600 \mathrm{ppmCH}$ & $\begin{array}{l}1,220(1,080-1,390) \\
1,430(1,280-1,620)\end{array}$ & $\begin{array}{r}7 \\
12\end{array}$ & $\begin{array}{l}\text { 末 } \\
\text { 已 }\end{array}$ & $\begin{array}{l}148.3 \\
142.6\end{array}$ \\
\hline
\end{tabular}

表 2 谷氨酰胺和天门冬酰胺对幼胚生长的影响

1964 年第 7 次试验,培养 21 天

\begin{tabular}{|c|c|c|c|c|}
\hline 培 养 基 & 接种时胚长 (微米) & 胚 数 & 接种时已否分化 & 21 天后增长 $(\%)$ \\
\hline 对照(WM) & $\begin{array}{l}1,180(1,040-1,270) \\
1,410(1,350-1,520)\end{array}$ & $\begin{array}{r}23 \\
7\end{array}$ & $\begin{array}{l}\text { 末 } \\
\text { 巳 }\end{array}$ & $\begin{array}{l}105.2 \\
106.3\end{array}$ \\
\hline $\mathrm{WM}+200 \mathrm{ppm}$ 谷管跣胺 $(\mathrm{Gl})$ & $\begin{array}{l}1,150(1,080-1,310) \\
1,420(1,340-1,540)\end{array}$ & $\begin{array}{r}19 \\
5\end{array}$ & $\begin{array}{l}\text { 未 } \\
\text { 已 }\end{array}$ & $\begin{array}{l}134.5 \\
143\end{array}$ \\
\hline $\mathrm{WM}+400 \mathrm{ppmGl}$ & $\begin{array}{l}1,160(1,040-1,310) \\
1,390(1,360-1,400)\end{array}$ & $\begin{array}{r}11 \\
7\end{array}$ & $\begin{array}{l}\text { 末 } \\
\text { 已 }\end{array}$ & $\begin{array}{l}155.6 \\
141.0\end{array}$ \\
\hline$W M+800 p p m G l$ & $\begin{array}{l}1,200(1,080-1,320) \\
1,430(1,390-1,540)\end{array}$ & $\begin{array}{r}14 \\
6 \\
\end{array}$ & $\begin{array}{l}\text { 末 } \\
\text { 已 }\end{array}$ & $\begin{array}{l}105.4 \\
106.3\end{array}$ \\
\hline $\mathrm{WM}+200 \mathrm{ppm}$ 天门冬酰胺(As) & $\begin{array}{l}1,190(1,040-1,310) \\
1,460(1,440-1,500)\end{array}$ & $\begin{array}{r}17 \\
5\end{array}$ & $\begin{array}{l}\text { 末 } \\
\text { 已 }\end{array}$ & $\begin{array}{l}100.8 \\
127.6\end{array}$ \\
\hline$W M+400 p p m A s$ & $1,190(1,040-1,320)$ & 21 & 未 & 102.9 \\
\hline $\mathrm{WM}+800 \mathrm{ppmAs}$ & $\begin{array}{l}1,180(1,040-1,320) \\
1,440(1,390-1,540)\end{array}$ & $\begin{array}{r}19 \\
3\end{array}$ & $\begin{array}{l}\text { 末 } \\
\text { 已 }\end{array}$ & $\begin{array}{l}77.1 \\
82.6\end{array}$ \\
\hline
\end{tabular}

从表 2 可以看出, $200-400 \mathrm{ppm}$ 谷氨酰 胺促进幼胚长度生长, 而 $800 \mathrm{ppm}$ 却沒有什 么作用。天门冬酰胺较低浓度（200-400 $\mathrm{ppm}$ ) 对幼胚生长看不出什么影响, 而较高 浓度 $(800 \mathrm{ppm})$ 则明孟抑制长度生长. 天门 冬酰胺对幼胚分化还具有明显的抑制作用.
$200 \mathrm{ppm}$ 天门冬酰胺使大部分幼胚不能正 常 分化子叶， $800 \mathrm{ppm}$ 抑制全部幼胚的子叶分 化. 试验曾重复两次, 结果基本一致.

\section{2,4-D 对幼肧生长的影响}

幼胚对 2,4-D 单独或与栁子乳汁一起处 理时的反应如表 3 所示. 
表 3 2,4-D 对幼胚生长的影响

1964 年第 12 大试验,培养 14 天

\begin{tabular}{|c|c|c|c|c|c|c|}
\hline 养 & 接种时胚长 ( 微米) & 胚数 & $\begin{array}{c}\text { 14天后增 } \\
\text { 长 }(\%)\end{array}$ & 态 & 特 & 征 \\
\hline 对照(WM) & $1,260(1,120-1,430)$ & 20 & 79.3 & \multicolumn{3}{|l|}{ 正常分化和生长 } \\
\hline $\mathrm{WM}+1_{\mathrm{ppm}} 2,4-\mathrm{D}$ & $1,300(1,160-1,430)$ & 13 & 98.4 & \multicolumn{3}{|c|}{ 正常分化; 一小部分稍长愈伤组织 } \\
\hline $\mathrm{WM}+6 \mathrm{ppm} 2,4-\mathrm{D}$ & $1,280(1,160-1,400)$ & 17 & 89.0 & \multicolumn{3}{|c|}{ 全部不分化; 约牛数秾长愈伤组织 } \\
\hline$W M+20 \%$ 楜子乳汁 $(\mathrm{CM})$ & $1,300(1,160-1,430)$ & 23 & 120.0 & \multicolumn{3}{|c|}{9 个胚多子叶；胚较肥厚 } \\
\hline $\mathrm{WM}+\mathrm{CM}+1 \mathrm{ppm} 2,4-\mathrm{D}$ & $1,260(1,160-1,430)$ & 22 & 131.7 & \multicolumn{3}{|c|}{ 10个胚多子叶; 稍长愈伤组织; 胚较肥厚 } \\
\hline $\mathrm{WM}+\mathrm{CM}+6 \mathrm{ppm} 2,4-\mathrm{D}$ & $1,270(1,160-1,430)$ & 27 & 123.5 & \multicolumn{3}{|c|}{ 全部不分化; 稍长愈伤组织; 胚较肥厚 } \\
\hline
\end{tabular}

表 4 硫酸腺尿園对幼胚生长的影响

1964 年第 10 大试验A 及 H 组, 培美 8 天

\begin{tabular}{|c|c|c|c|c|c|c|}
\hline 培 & 养 & 基 & 接种时胚长 (微米) & 胚 & 数 & 8 天后增长 $(\%)$ \\
\hline \multicolumn{3}{|c|}{ 对照（WM） } & $1,255(1,160-1,390)$ & & & 57.7 \\
\hline \multicolumn{3}{|c|}{$\mathrm{WM}+40 \mathrm{ppm}$ 硫酸腺尿㶅 } & $1,225(1,080-1,390)$ & 1 & & 61.3 \\
\hline
\end{tabular}

从表 3 可以看出, 2,4-D 对幼胚仅有微 弱的促进作用，但 $6 \mathrm{ppm} 2,4-\mathrm{D}$ 明显抑制幼 胚的分化. 此外, 2,4-D 还引起轻微愈伤组 织的产生. 在基本培养基中加入 $20 \%$ 楖子 乳汁，2,4-D 的作用仍明显， $1 \mathrm{ppm}$ 沒有影 响，6ppm 抑制分化，由于楖子乳汁的作用， 胚体都长得比较肥大. 从培养胚的制片也可 以看出，2,4-D (5ppm 以上) 抑制子叶和苗 端分生组织的形成. 试验曾重复 4 次, 结果 基本一致.

\section{4. 硫酸腺尿園对幼胚生长的影响}

在基本培养基中加入 $400 \mathrm{ppm}$ 腺尿園， 培养 8 天后所得测量数据如表 4 所示.

从表 4 可以看出, $40 \mathrm{ppm}$ 硫酸腺尿園对 幼胚生长沒有什么影响. 加 $20 \mathrm{ppm}$ 或 $40 \mathrm{ppm}$ 硫酸腺尿園的胚与对照的胚相比，在形态上 沒有什么区別，只是在加硫酸腺尿園培养的 胚, 胚根端颜色变黑, 看起来显得小一些. 在 基本培养基中加 $20 \%$ 楖子孚汁,再加 $20 \mathrm{ppm}$ 或 $40 \mathrm{ppm}$ 硫酸腺尿圓培养的胚，与不加的 也沒有区別. 这三组的胚都长得比较肥厚,
完全是楖子孚汁的作用. 试验曾重复一次, 结果一致.

\section{讨 论 和 结 论}

酪朊水解物对大麦胚 ${ }^{[4,5]}$ 、荠荣胚 ${ }^{[6]}$ 、棉 胚 ${ }^{[7]} 、$ Citrus microcarpa 胚 ${ }^{[8]} 、$ Dendrophthoe falcata 胚 ${ }^{[9]}$ 在离体培养中都有促进生长的作 用. 银杏的试验也说明酪朊水解物对幼胚生 长有促进作用，但它的作用显然不如楖子乳 汁 $(20 \%)^{[10]}$, 同时与 Ranga Swamy ${ }^{[8]}$ 在 $\mathrm{Ci}$ trus microcarpa 离体试验中所得到的结果不 同.

Rijven ${ }^{[6,11,12]}$ 报告过谷氨酰胺对荠荣及其 他十多种植物胚的生长刺激作用. 谷氨酰胺 对银杏成熟胚 ${ }^{[13]}$ 、大麦幼胚 ${ }^{[14]}$ 、向日葵幼 胚 ${ }^{[15]}$ 的离体生长都有刺激作用, 而天门冬酰 胺 $(400 \mathrm{ppm})$ 对幼胚却有抑制作用 ${ }^{[11,12]}$. 银杏 的试验结果表明谷氨酰胺促进幼胚生长, 而 天门冬酰胺 (200- $800 \mathrm{ppm})$ 却明显抑制胚的 分化.

$$
\text { 2, 4-D (1-2ppm) 诱导黑麦胚愈伤组织 }
$$


的产生和持续生长 ${ }^{[2]}$. 目前的试 验 表 明, 1ppm 2,4-D 对幼胚生长沒有什么影响，而 $5 \mathrm{ppm}$ 以上 2,4-D 则明显抑制幼胚分化. 由 此可见， 2,4-D 有控制形态发生的机能.

银杏的试验还表明，20- $40 \mathrm{ppm}$ 硫酸腺 尿園对幼胚生长沒有什么影响.

根据银杏离体幼胚的试验结果，可以得 到下面几点结论:

1. 酪朊水解物有促进幼胚生长的作用, 以 $400 \mathrm{ppm}$ 浓度发育较为正常，但效果不及 楖子孚乚汁 $(20 \%)$, 较高浓度常诱导愈伤组织 的产生.

2. 谷氨酰胺 (200-400 ppm) 促进幼胚生 长, 而天门冬酰胺 $(200-800 \mathrm{ppm})$ 抑制幼胚 分化, $800 \mathrm{ppm}$ 抑制胚长度生长.

3. 1 ppm 2,4-D 对幼胚生长沒有什么影 响, 较高浓度 (5ppm 以上) 完全抑制原胚分 化.

4. 高浓度硫酸腺尿圓 $(20-40 \mathrm{ppm})$ 对幼 胚生长沒有什么影响。

\section{参考 文 献}

[1] Narayanaswami, S. and Norstog, K., Bot. Rev., 30[4], 587-628 (1964).

[2] Carew, D. P. and Schwarting, A. E., Bot. Gaz., 119, 237-239 (1958).

[3] 王伏雄、陈㸖钘、李宪章, 相物学报, 11, 217-224 (1963).

[4] Kent, N. and Brink, R. A., Science, 106, 547 (1947).

[5] Ziebur, N. K., Brink, R. A., Graf, L. H. and Stahmann, M. H., Amer. Jour. Bot., 37, 144148 (1950).

[6] Rijven, A. H. G. C., Acta Bot. Néerl., 1, 157200 (1952).

[7] Mauney, J. R., Bot. Gaz., 122, 205-209 (1961).

[8] Ranga Swamy, N. S., Phytomorphology, 11, 109129 (1961).

[9] Johri, B. M. and Singh Bajaj, Y. P., Plant tissuc and organ culture -A symposium, 292-301, 1963.

[10] 王伏雄、陈祖镊, 植物学报, 13, 224-239 (1965).

[11] Rijven, A. H. G. C., Koninkl. Nederl. Akad.van Wetens. Proc. Ser., C 58, 368-376 (1955).

[12] Rijven, A. H. G. C., Aust. Jour. Biol. Sci., 9, 511-527 (1956).

[13] Ball, E., Amer. Jour. Bot., 46, 130-139 (1959).

[14] Norstog, K. and Smith, J. E., Science, 142 (3600), 1655-1656 (1963).

[15] 郭仲垛、王伏雄, 植物学报, 12, 364-376 (1964). 